\title{
Terahertz Time-Domain Polarimetry of Carbon Nanomaterials
}

\author{
Anatoly Kvitsinskiy ${ }^{1,2}$, Petr Demchenko ${ }^{1}$, Alexander Grebenchukov ${ }^{1,2,3,4}$, Egor Litvinov ${ }^{1}$, \\ Maxim Masyukov ${ }^{1,3}$, Anton Zaitsev ${ }^{1}$, Ilya Anoshkin ${ }^{1}$, Anna Baldycheva ${ }^{5,6}$, Evgeniya Kovalska ${ }^{6}$, \\ Anna Vozianova ${ }^{1}$, and Mikhail Khodzitsky ${ }^{1,3}$ \\ ${ }^{1}$ Terahertz Biomedicine Laboratory, ITMO University, Saint Petersburg 199034, Russian Federation \\ ${ }^{2}$ Center for Bioengineering, ITMO University, Saint Petersburg 197101, Russian Federation \\ ${ }^{3}$ Radiation Medicine Laboratory, ITMO University, Saint Petersburg 199034, Russian Federation \\ ${ }^{4}$ Optoelectronics and Measurements Techniques Laboratory, University of Oulu, Oulu 90570, Finland \\ ${ }^{5}$ College of Engineering, Mathematics and Physical Sciences, University of Exeter, Exeter EX4 4QF, UK \\ ${ }^{6}$ Graphene Centre, University of Exeter, Exeter EX4 4QL, UK
}

\begin{abstract}
Terahertz time-domain spectroscopic polarimetry (THz-TDSP) method was used to study of polarization properties of a few-layer graphene (FLG) and a randomly oriented singlewalled carbon nanotube (SWCNT) thin film on silicon (Si) substrates in terahertz ( $\mathrm{THz})$ frequency range under an external optical pumping (OP) and an external static magnetic field (MF). Frequency dependencies of azimuth and ellipticity angles of a polarization ellipse of the samples were obtained experimentally. The results confirm the fact that, based on carbon nanomaterials, it is possible to devise tunable $\mathrm{THz}$ polarization modulators for use in the latest security and telecommunication systems.
\end{abstract}

\section{INTRODUCTION}

$\mathrm{T}$ ERAHERTZ $(\mathrm{THz})$ radiation is widely used in physics and astronomy, chemistry and medicine, security and telecommunication systems, and other fields of science and technology [1]. Currently, an important area of $\mathrm{THz}$ photonics is study of multilayer graphene [2] and carbon nanotubes [3] under an external optical pumping (OP) and an external static magnetic field to devise efficient tunable $\mathrm{THz}$ polarization modulators based on them [4], [5]. One of the main methods for studying polarization and magneto-optical properties of carbon nanomaterials is the $\mathrm{THz}$ time-domain spectroscopic polarimetry (THz-TDSP) [6].

\section{Material AND Methods}

The few-layer graphene (FLG) with 10 layers of graphene, the randomly oriented single-walled carbon nanotube (SWCNT) thin film on silicon ( $\mathrm{Si}$ ) substrates and a sample of the Si substrate were studied. FLG was synthesized on a nickel substrate by the chemical vapor deposition [7] in quartz furnace and then transferred to a $\mathrm{Si}$ substrate. SWCNT thin film was synthesized on a nitrocellulose micropore filter by the catalytic ethanol-chemical vapor deposition [8] and then transferred to a $\mathrm{Si}$ substrate. SWCNTs diameters were calculated using the Kataura plot [9] and were 1.3-2.0 nm. SWCNTs length was visualized by the transmission electron microscopy [10] and was $\sim 15 \mu \mathrm{m}$. SWCNT film thickness was calculated from the optical absorbance at $550 \mathrm{~nm}$ [11] and was $\sim 110 \mathrm{~nm}$. Si substrate thickness was $\sim 1 \mathrm{~mm}$. The samples were studied using the THz-TDSP system based on the $\mathrm{THz}$ time-domain spectrometer [12], two polarizers, $980 \mathrm{~nm}$ laser for creating an external $\mathrm{OP}$ of $\sim 1.0 \mathrm{~W} \cdot \mathrm{cm}^{-2}$ and $\mathrm{NdFeB}$ axially magnetized magnet for creating an external static MF of $\sim 1.3 \mathrm{~T}$. Scheme of the experimental THz-TDSP setup and pictures of the experimental samples are shown in Fig. 1.
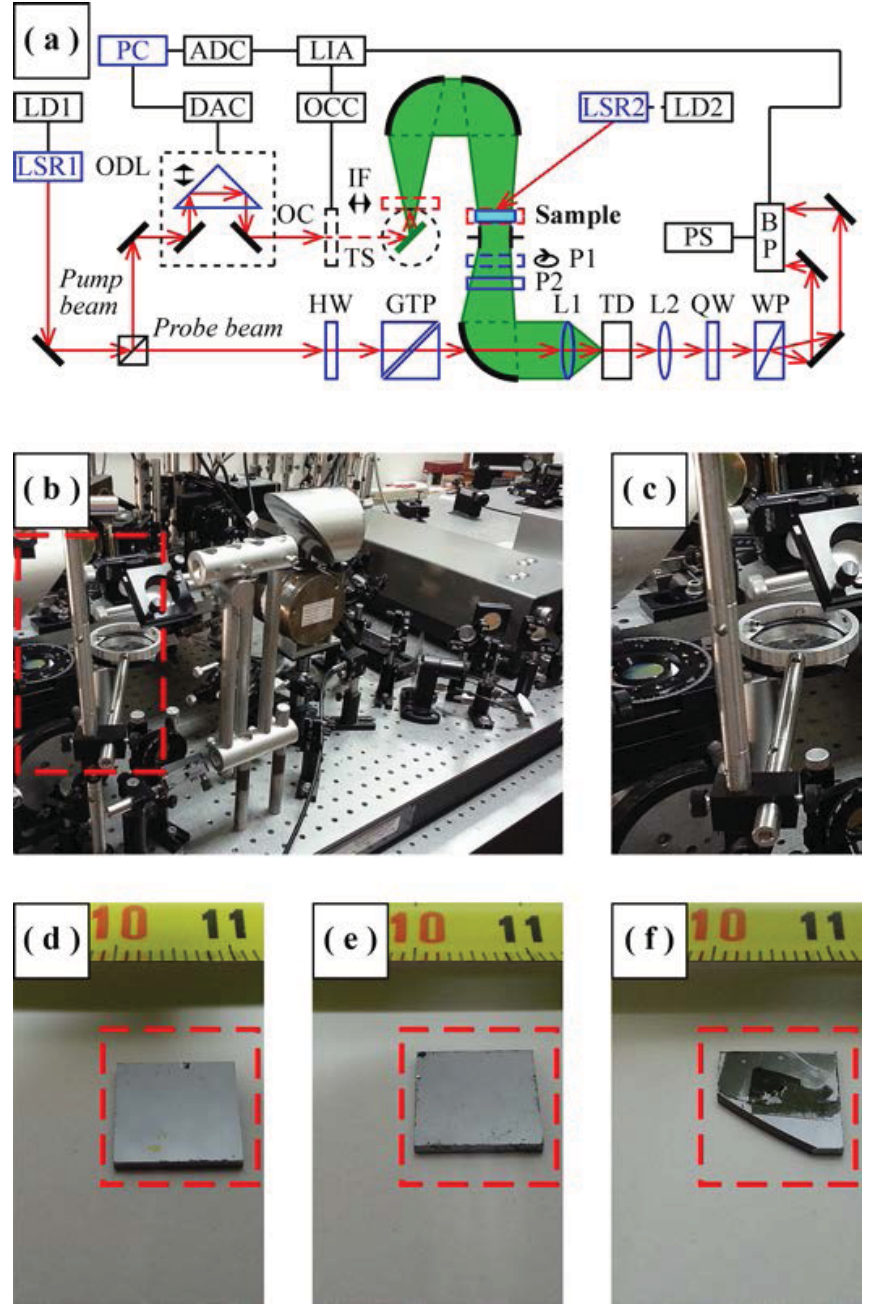

experimental THz-TDSP setup (ADC - analog-to-digital converter; BP balanced photodetector; DAC - digital-to-analog converter; GTP - GlanTaylor prism; $\quad$ HW - half-wave IF - infrared cut-off filter; L1, L2 - positive lenses; LD1, LD2 - laser diodes' drivers; LIA - lock-in amplifier; LSR1 - Yb:KYW 1040 nm laser; LSR2 - $980 \mathrm{~nm}$ laser; OC - optical chopper; OCC - optical chopper controller; ODL - optical delay line; P1 - rotating polarizer; P2 - static polarizer; PC - personal computer; PS - balanced detector power supply; QW - quarter-wave plate; TD - THz radiation detector based on CdTe crystal; TS - THz radiation source based on InAs crystal; WP - Wollaston prism); (b) picture of the assembled experimental THz-TDSP setup; (c) picture of a cuvette for the experimental samples; (d) picture of the $\mathrm{Si}$ substrate (highlighted in red); (e) picture of the FLG on the Si substrate (in red); and (f) and picture of the SWCNT thin film on the Si substrate (in red). 


\section{RESULTS}

For each sample, temporal waveforms of the transmitted $\mathrm{THz}$ signals under various external influences were recorded using LabVIEW software at parallel and crossed by $45^{\circ}$ positions to the transmission direction of the polarizers. Experimental data processing was done using MATLAB software, 4th order coiflet-based denoising technique and rectangular signal windowing [13]. The windowing was done to exclude the influence of the water vapor absorption. Frequency dependencies of the azimuth $\psi$ and the ellipticity $\chi$ angles of the polarization ellipse of the samples were calculated from the Stokes parameters [14]:

$$
\begin{aligned}
& \left(\begin{array}{l}
S_{0} \\
S_{1} \\
S_{2} \\
S_{3}
\end{array}\right)=\left(\begin{array}{c}
E_{1}^{2}+E_{2}^{2} \\
E_{1}^{2}-E_{2}^{2} \\
2 \cdot E_{1} \cdot E_{2} \cdot \cos \delta \\
2 \cdot E_{1} \cdot E_{2} \cdot \sin \delta
\end{array}\right) \\
& \left(\begin{array}{l}
\psi \\
\chi
\end{array}\right)=\left[\begin{array}{l}
0.5 \cdot \sin ^{-1}\left(S_{3} / S_{0}\right) \\
0.5 \cdot \tan ^{-1}\left(S_{2} / S_{1}\right)
\end{array}\right]
\end{aligned}
$$

where $E_{1}$ and $E_{2}$ are amplitudes of parallel and perpendicular components of electric field $\boldsymbol{E}$ vector, and $\delta$ is the phase difference between them. The results are shown in Fig. 2-4.
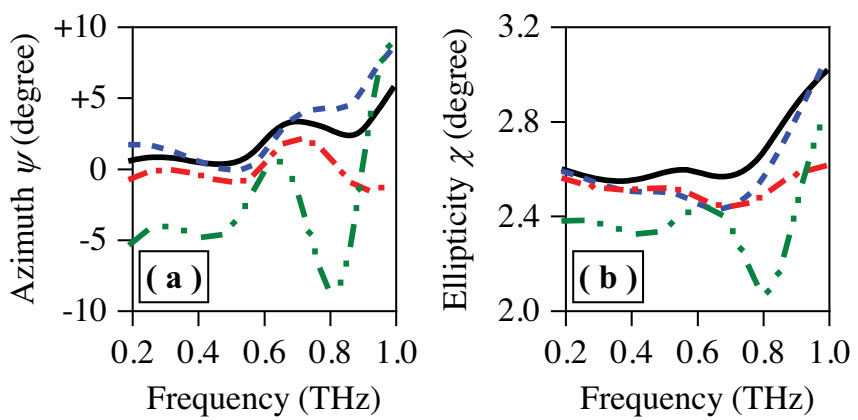

$$
\begin{array}{ll}
-\mathrm{Si} & =-\mathrm{Si}, \mathrm{OP}\left(\sim 1.0 \mathrm{~W} / \mathrm{cm}^{2}\right) \\
=--\mathrm{Si}, \mathrm{MF}(\sim 1.3 \mathrm{~T})-\cdots \mathrm{Si}, \mathrm{OP}, \mathrm{MF}
\end{array}
$$

Fig. 2. Frequency dependences of (a) azimuth $\psi$ and (b) ellipticity $\chi$ angles of the polarization ellipse of the $\mathrm{Si}$ substrate.Fig. 1. (a) Scheme of the
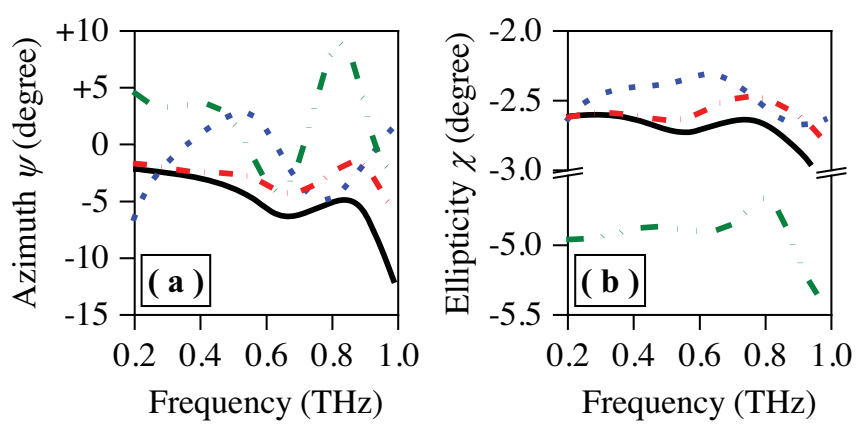

$$
\begin{array}{ll}
-\mathrm{FLG} & -=-\mathrm{FLG}, \mathrm{OP}\left(\sim 1.0 \mathrm{~W} / \mathrm{cm}^{2}\right) \\
-\cdots-\mathrm{FLG}, \mathrm{MF}(\sim 1.3 \mathrm{~T})-\cdots & \mathrm{FLG}, \mathrm{OP}, \mathrm{MF}
\end{array}
$$

Fig. 3. Frequency dependences of (a) azimuth $\psi$ and (b) ellipticity $\chi$ angles of the polarization ellipse of the FLG without the Si substrate.

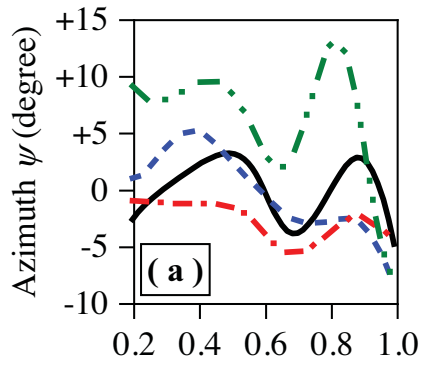

Frequency $(\mathrm{THz})$

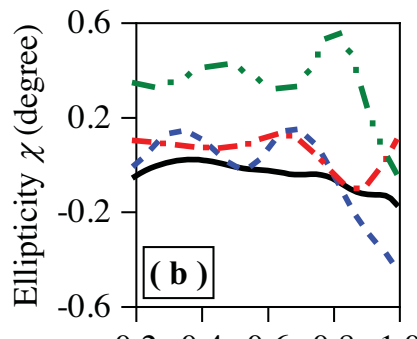

Frequency $(\mathrm{THz})$

$$
\begin{aligned}
& \text { - SWCNT - - - -SWCNT, OP }\left(\sim 1.0 \mathrm{~W} / \mathrm{cm}^{2}\right) \\
& \text { - - - SWCNT, MF ( 1.3 T) - - . SWCNT, OP, MF }
\end{aligned}
$$

Fig. 4. Frequency dependences of (a) azimuth $\psi$ and (b) ellipticity $\chi$ angles of the polarization ellipse of the SWCNT thin film without the Si substrate.

\section{SUMMARY}

The changes in the angles are the result of the magnetooptical Faraday effect. Applying MF to an isotropic material cause anisotropy, and as a result a circular birefringence. Taking this into account, the vector of the transmitted linearly polarized wave is not the same as it was before the sample. The azimuth polarization angle rotation caused by the Faraday effect depends on free carrier concentration, which can be increased by OP. The results confirm the fact that, based on nanomaterials, it is possible to devise efficient tunable $\mathrm{THz}$ polarization modulators for telecommunication systems.

This work was supported by RSF (Grant №19-72-10141).

\section{REFERENCES}

[1]. S.S. Dhillon etal., "The 2017 terahertz science and technology roadmap," J. Phys. D. Appl. Phys., vol. 50, no. 4, p. 043001, Jan. 2017.

[2]. A. N. Grebenchukov etal., "Faraday effect control in graphenedielectric structure by optical pumping," J.Magn.Magn.Mater., vol.472, pp. 25-28, Feb. 2019.

[3]. S. Smirnov et al., "Optically controlled dielectric properties of singlewalled carbon nanotubes for terahertz wave applications," Nanoscale, vol. 10, no. 26, pp. 12291-12296, Jun. 2018.

[4]. J.Shi etal., "THz photonics in two dimensional materials and metamaterials: properties, devices and prospects," J.Mater. Chem. C.Mater., vol. 6, no. 6, pp. 1291-1306, Jan. 2018.

[5]. R. Wang et al., "Mechanisms and applications of carbon nanotubes in terahertz devices: a review," Carbon, vol. 132, pp. 42-58, Jun. 2018.

[6]. G. Zhao et al., "A dual-port THz time domain spectroscopy system optimized for recovery of a sample's Jones matrix," Sci.Rep., vol.9, Feb. 2019, Art no. 2099.

[7]. E. Kovalska et al., "Multi-layer graphene as a selective detector for future lung cancer biosensing platforms," Nanoscale, vol.11, no. 5, pp. 2476-2483, Jan. 2019.

[8]. A. A. Tonkikh et al., "Single-wall carbon nanotube film grown by advanced ethanol chemical vapor deposition process," J. Nanoelectron. Optoelectron., vol. 7, pp. 99-101, Jan. 2012.

[9]. H. Kataura et al., "Optical properties of single-wall carbon nanotubes," Synth. Met., vol. 103, no. 1-3, pp. 2555-2558, Jun. 1999.

[10]. B. Krause et al., "A method for determination of length distributions of multiwalled carbon nanotubes before and after melt processing," Carbon, vol. 49, pp. 1243-1247, Apr. 2011.

[11]. A. G. Nasibulin et al., "Multifunctional free-standing single-walled carbon nanotube films," ACS Nano, vol. 5, pp. 3214-3221, Mar. 2011.

[12]. V. G. Bespalov et al., "Methods of generating superbroadband terahertz pulses with femtosecond lasers," J. Opt.Technol., vol. 75, pp.636-642, Oct. 2008 .

[13]. B. Ferguson et al., "De-noising techniques for terahertz responses of biological samples," Microelectronics J., vol. 32, pp. 943-953, Dec. 2001. [14]. M. Born and E. Wolf, Principles of Optics, 7th ed. Cambridge, UK:
Cambridge Univ. Press, 2005 . 